\title{
Reliability of digital ulcer definitions as proposed by the UK Scleroderma Study Group: A challenge for clinical trial design
}

Journal of Scleroderma and Related Disorders 2018, Vol. 3(2) 170-174 (C) The Author(s) 2018 Reprints and permissions: sagepub.co.uk/journalsPermissions.nav DOI: $10.1177 / 2397198318764796$ journals.sagepub.com/home/jso (SAGE

\author{
Michael Hughes', Andrew Tracey', Monica Bhushan', \\ Kuntal Chakravarty ${ }^{3}$, Christopher P Denton ${ }^{3}$, Shirish Dubey, \\ Serena Guiducci ${ }^{5}$, Lindsay Muir ${ }^{6}$, Voon Ong $^{3}$, Louise Parker ${ }^{3}$, \\ John D Pauling ${ }^{7}$, Athiveeraramapandian Prabu ${ }^{8}$, Christine Rogers9, \\ Christopher Roberts ${ }^{10}$ and Ariane L Herrick ${ }^{1,11}$
}

\begin{abstract}
Introduction: The reliability of clinician grading of systemic sclerosis-related digital ulcers has been reported to be poor to moderate at best, which has important implications for clinical trial design. The aim of this study was to examine the reliability of new proposed UK Scleroderma Study Group digital ulcer definitions among UK clinicians with an interest in systemic sclerosis.

Methods: Raters graded (through a custom-built interface) 90 images (80 unique and 10 repeat) of a range of digital lesions collected from patients with systemic sclerosis. Lesions were graded on an ordinal scale of severity: 'no ulcer', 'healed ulcer' or 'digital ulcer'.

Results: A total of 23 clinicians - 18 rheumatologists, 3 dermatologists, I hand surgeon and I specialist rheumatology nurse - completed the study. A total of 2070 (1840 unique +230 repeat) image gradings were obtained. For intra-rater reliability, across all images, the overall weighted kappa coefficient was high $(0.7 \mathrm{I})$ and was moderate $(0.55)$ when averaged across individual raters. Overall inter-rater reliability was poor (0.15).

Conclusion: Although our proposed digital ulcer definitions had high intra-rater reliability, the overall inter-rater reliability was poor. Our study highlights the challenges of digital ulcer assessment by clinicians with an interest in systemic sclerosis and provides a number of useful insights for future clinical trial design. Further research is warranted to improve the reliability of digital ulcer definition/rating as an outcome measure in clinical trials, including examining the role for objective measurement techniques, and the development of digital ulcer patient-reported outcome measures.
\end{abstract}

\section{Keywords}

Systemic sclerosis, scleroderma, digital ulcers, clinical trials, outcome measures

Date received: 2 January 20I8; accepted: 21 February 2018.

\footnotetext{
'Centre for Musculoskeletal Research, The University of Manchester, Salford Royal NHS Foundation Trust, Manchester Academic Health Science Centre, Manchester, UK

${ }^{2}$ Department of Dermatology, Blackpool Teaching Hospitals NHS Foundation Trust, Clifton Hospital, Lytham St Annes, UK

${ }^{3}$ Centre for Rheumatology and Connective Tissue Diseases, Royal Free Hospital, London, UK

${ }^{4}$ Department of Rheumatology, University Hospitals Coventry and Warwickshire NHS Trust, Coventry, UK

${ }^{5}$ Department of Experimental and Clinical Medicine, University of Florence, Florence, Italy

${ }^{6}$ Department of Hand Surgery, Salford Royal NHS Foundation Trust, Salford, UK

${ }^{7}$ Department of Pharmacy \& Pharmacology, University of Bath, Bath, UK
}

\footnotetext{
${ }^{8}$ Rheumatology Department, Birmingham City Hospital, Sandwell and West Birmingham Hospitals NHS Trust, Birmingham, UK

${ }^{9}$ The University of Manchester, Manchester, UK

${ }^{10}$ Centre for Biostatistics, Institute of Population Health, School of Medicine, The University of Manchester, Manchester, UK

IINIHR Manchester Biomedical Research Centre, Central Manchester NHS Foundation Trust, Manchester Academic Health Science Centre, Manchester, UK
}

Corresponding author:

Michael Hughes, Centre for Musculoskeletal Research, The University of Manchester, Salford Royal NHS Foundation Trust, Manchester Academic Health Science Centre, Manchester M6 8HD, UK. Email: michael.hughes-6@postgrad.manchester.ac.uk 
Table I. Proposed UKSSG working group DU definitions.

Digital ulcer A lesion (on the finger or distal to the metacarpophalangeal joint) with loss of surface epithelisation and a visually
discernible depth. The ulcer bed is often wet in appearance with surface slough.
The peri-lesional skin surrounding digital ulcers is not uncommonly erythematous and/or macerated (including
in the absence of superadded infection). Patients often report pain (which may be severe) associated with
digital ulcers. Digital ulcers often have an overlying scab (eschar), and if there is a high index of suspicion of an
underlying digital ulcer, then the lesion should be classified as such. Common sites for digital ulcers include the
fingertips and over the extensor (dorsal) aspects of the hands and in relation to subcutaneous calcinosis. Less
often digital ulcers may occur at other sites on the hands (e.g. over the lateral aspects of the digits and at the
base of the nail).
A lesion with complete surface epithelisation (otherwise the lesion would be classified as a 'digital ulcer').
No ulcer ulcer
Any lesion which does not fulfil the definitions of either a 'digital ulcer' or 'healed ulcer' including (but not limited
to) digital pitting scars, hyperkeratosis, and fissures.

\section{Introduction}

The reliability of rheumatologists grading digital ulcers (DUs) in patients with systemic sclerosis (SSc) has been reported to be poor to moderate at best,,$^{1-3}$ which is a major concern in the design of future clinical trials. Despite a number of drug therapies ${ }^{4-7}$ to prevent and treat incident DUs, recurrent DUs remain a major source of pain and disability in some patients with SSc. ${ }^{8}$ There is a strong unmet clinical need to broaden therapeutic options to reduce the burden of SSc-DU disease, underpinning the need for high-quality clinical trials.

Recent multi-centre clinical trials of drug therapies for DUs have used different definitions for DUs in their study design. In general, previous definitions ${ }^{5,7,9,10}$ have included a loss of surface epithelisation and with a discernible depth. Many studies have only included those DUs which occur on the fingertips, as these are considered 'ischaemic' and therefore presumably most likely to respond to vascular therapies, excluding those which occur over the extensor aspect of the fingers. There are also a number of digital lesions (e.g. pitting scars and fissures) which are common in patients with SSc, and can be very challenging to distinguish from DUs. Furthermore, the inclusion of DUs in the current American College of Rheumatology (ACR)/ European League Against Rheumatism (EULAR) SSc classification criteria ${ }^{11}$ highlights the importance of accurate definition of DUs in patients with SSc.

Against this background, a UK Scleroderma Study Group (UKSSG) working group was convened to develop and test new DU definitions. The aim of this study was to examine the reliability of the proposed DU definitions among UK clinicians with an interest in SSc.

\section{Methods}

\section{UKSSG working group}

Under the auspices of the UKSSG, a working group was assembled comprising eight UK-based rheumatologists with an interest in SSc, an international SSc expert, a dermatologist, a hand surgeon and a rheumatology specialist nurse. A statistician with extensive experience of reliability research and two patients with SSc and a history of DUs were also members of the working group.

\section{Consensus meeting and derivation of proposed DU definitions}

A DU consensus meeting was convened at the University of Manchester on 24 November 2015. Previous DU definitions and issues around the challenges of DU grading were discussed. A key issue that emerged from the meeting was that different outcome measures might be required for preventive studies versus those studies investigating treatments for DUs. After the meeting, based upon the discussions, proposed DU definitions were drafted. These were then sent to the members of the working group for comment before being finalised. The final definitions for 'no ulcer', 'healed ulcer' and 'DU' are presented in Table 1.

\section{Study design and participants}

A total of 80 clinical images of a range of digital lesions (mainly DUs) from our previous reliability study ${ }^{3}$ were used to conduct the grading exercise. These were prospectively selected by two individuals (M.H. and A.L.H.) to encompass the range of digital lesions observed in patients with SSc-spectrum disorders. A gangrenous digit was specifically included, as this is a controversial issue in the definition of DUs. As previously described, ${ }^{3}$ a clinical photograph of the digital lesion was obtained by a trained medical photographer, with a $1 \mathrm{~cm}$ graded scale positioned in close proximity to the lesion, to give raters an indication of the lesion size. Patient and lesion characteristics have been previously reported. ${ }^{3}$ The study was approved by the National Research Ethics Committee East of EnglandHatfield, and all patients provided signed informed consent.

A new custom-built, secure web-based interface was constructed to both display and record the grading of the clinical images. All clinical members of the UKSSG (and members of the working group) were invited to participate 


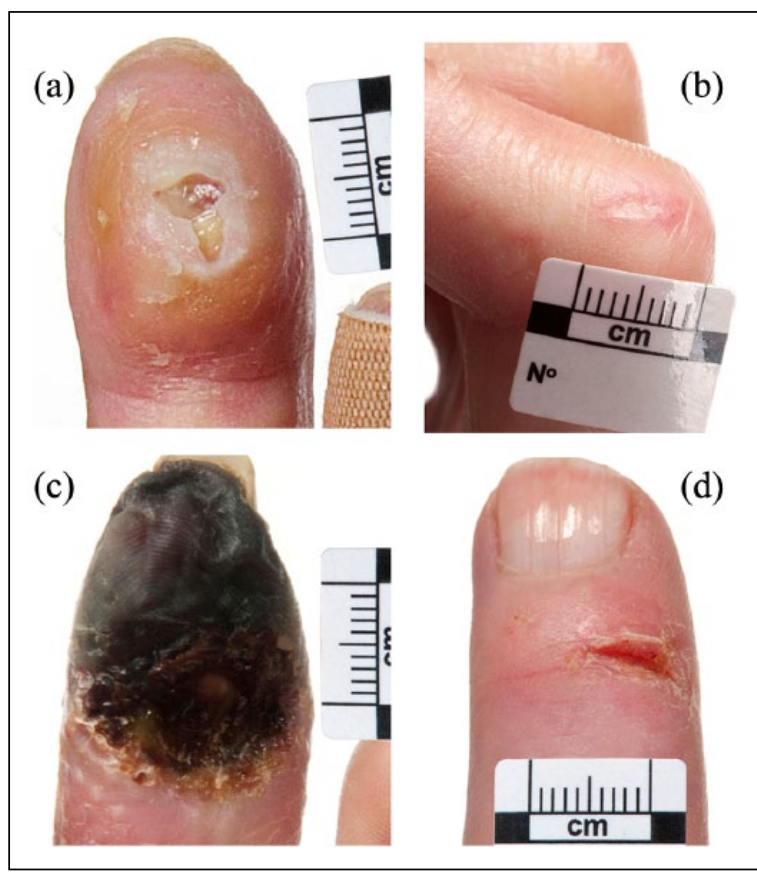

Figure I. Example images of the proposed UKSSG DU definitions demonstrating different degrees of agreement among raters. (a) High agreement (23 'DU'). (b) High agreement (3 'no ulcer', 20 'healed ulcer' and 0 'DU'). (c) Low agreement ( 10 'no ulcer', 0 'healed ulcer' and I 3 'DU'). (d) Low agreement ( 16 'no ulcer', 0 'healed ulcer' and 7 'DU').

in the web-based study. On the 'entry' screen, the proposed DU definitions were presented without any exemplar images. The definitions could be recalled for review throughout each rater's participation in the study. Each rater graded 90 images: 80 unique and then 10 repeated images ('randomly' selected from the first 50) to allow an assessment of intra-rater reliability. The unique images were displayed in a randomised order to each rater. Raters graded each image according to the proposed definitions on a 3-point ordinal scale of severity: either 'no ulcer' $(0)$, 'healed ulcer' (1) or 'DU' (2). Raters had only one opportunity to score the image before choosing to move onto the next image.

\section{Statistical analysis}

The reliability of categorical data (here 'no ulcer', 'healed ulcer' and 'DU') can be assessed using kappa coefficients, which calculate the level of agreement between raters. Where the scale is ordered, a weighted kappa coefficient (which is also an intraclass coefficient) is used. Similar to our previous studies, ${ }^{1,3}$ intra-rater reliability was assessed using a weighted kappa coefficient with quadratic weights. This was calculated for each grader before taking the mean to obtain an overall figure. Inter-rater reliability assessment was based on the first observation of an image by a rater. One-way analysis of variance (ANOVA) was used to estimate and to assess overall inter-rater reliability, which gives an estimate of the kappa coefficient. ${ }^{12}$ Data were dichotomised by adjoining adjacent categories which could be considered as applicable to (a) 'preventive studies' (i.e. no ulcer vs healed ulcer and DU) and (b) 'studies of treatments for DUs' (i.e. no ulcer and healed ulcer vs DU). It has been suggested that the kappa can be interpreted as no better than chance alone $(<0)$, poor $(0.01-$ $0.20)$, fair $(0.21-0.40)$, moderate $(0.41-0.60)$, substantial (0.61-0.80), almost perfect (0.81-0.99), and perfect (1) agreement between raters. ${ }^{13}$ All statistical analyses on the data were performed using STATA, version 13.

\section{Results}

A total of 23 UK clinicians (raters) - 18 rheumatologists, 3 dermatologists, 1 orthopaedic hand surgeon and 1 specialist rheumatology nurse - successfully completed the study. A total of 2070 (1840 unique +230 repeat) image gradings were obtained.

\section{Intra-rater reliability}

The overall intra-rater reliability was good. Across all images $(n=230)$, irrespective of the individual rater, the overall weighted kappa $(\kappa)$ coefficient was 0.71 (95\% confidence interval $(\mathrm{CI})=0.63-0.79)$ and was moderate when averaged per individual raters $(\kappa=0.55$, standard deviation $(\mathrm{SD})=0.31)$. Intra-rater reliability was high for both the dichotomised analyses of 'no ulcer' versus 'healed ulcer and DU' $(\kappa=0.70,95 \% \mathrm{CI}=0.62-0.79)$ and 'no ulcer and healed ulcer' versus 'DU' $(\kappa=0.77,95 \% \mathrm{CI}=0.67-0.86)$.

\section{Inter-rater reliability}

The overall inter-rater reliability was poor $(\kappa=0.15,95 \%$ $\mathrm{CI}=0.10-0.21$ ). Inter-rater reliability was fair for the dichotomised analyses of 'no ulcer' versus 'healed ulcer' and 'DU' $(\kappa=0.25,95 \% \mathrm{CI}=0.19-0.31)$ and moderate for 'no ulcer and healed ulcer' versus 'DU' $(\kappa=0.41,95 \%$ $\mathrm{CI}=0.33-0.49)$.

Figure 1 illustrates a number of example images with high or low agreement between raters.

\section{Discussion}

The key finding of our study is that although our proposed UKSSG DU definitions had good intra-rater reliability, the agreement between raters was poor. This further confirms the urgent need to develop more reliable methods for the assessment of DUs as outcome measures in multi-centre, multi-rater studies.

The overall inter-rater reliability was lower than previously reported. ${ }^{1-3}$ In our previous web-based DU reliability study, ${ }^{3}$ the addition of 'real-world' (e.g. pain and discharge) 
clinical contextual information (which did not include information on previous debridements) did not significantly increase the inter-rater reliability $(\kappa=0.32$ without or 0.36 with the contextual information). The poor agreement between raters may be related to the intrinsic properties/ performance of our proposed definitions and/or to differences in rater opinion. The high intra-rater reliability further confirms the importance for the same individual to assess patients in clinical trials and to minimise the impact of differences in opinion between raters. The incorporation of centralised analysis of lesion images by a panel of trained experts might be one approach to ensuring uniformity in lesion assessment in clinical trials of SSc-DU.

A key strength of our study is that the definitions were developed by a broad working group including colleagues from related specialities (dermatology, orthopaedic hand surgery and rheumatology specialist nursing), with patient representation. Furthermore, a large image bank was used facilitating $>2000$ individual assessments, allowing comprehensive analysis of rater reliability.

Our UKSSG definitions can be considered as complementary to the recently proposed definitions by the World Scleroderma Foundation (WSF).${ }^{14}$ Both (sets of) definitions feature a loss of depth/epithelium as a central feature of DUs. In addition, both recognise that DUs are often covered by an overlying crust or eschar, and therefore, a caveat is added to both, and if debridement would likely confirm a DU, then the lesion should be classified as a DU. Neither set of definitions included an 'unclassifiable' category, as this was not felt to be helpful in the grading of DUs. In our definitions, we chose to encompass the spectrum of DU disease, including extensor DUs and those which occur in relation to subcutaneous calcinosis.

At present, assessment of treatment efficacy in clinical trials is primarily based upon clinician opinion alone: patient opinion has been less widely studied. In a recent reliability study, the agreement between individual patients and rheumatologists was poor with and without the clinical context ( 0.28 and 0.19 , respectively). ${ }^{3}$ The appearance of digital lesions does not always correlate with patient symptoms and there is a major unmet need to develop patient-reported outcome (PRO) instruments for capturing the multifaceted patient experience of SSc-DU to facilitate future clinical trials.

Our study has a number of important considerations. This was a web-based study and it could be argued that there is an important difference between assessing clinical photographs and physical examination of lesions. In our study, we chose not to provide exemplar images, because we wanted to assess the reliability of our proposed UKSSG definitions without the risk of biasing results (exemplar images could potentially have influenced rater grading). The inter-rater reliability of clinicians physically assessing digital lesions using the WSF definition was reported to be $0.5,{ }^{14}$ but the authors accepted that both the number of patients assessed and the number of clinicians who graded the lesions in this exercise was small (both $\mathrm{n}=7$ ). Similarly, the study by Baron et al., ${ }^{2}$ in which raters physically assessed DUs, included only a limited number $(n=10)$ of raters. It is unlikely that rater recall accounted for the high intra-rater reliability in this study due to the large number of images assessed and the systematic approach taken to repeat image assessment.

The limitations of clinician grading highlight the potential value of more objective methods for measuring DUs in future clinical trials, for example, ultrasound assessment of DU surface area and/or depth. In a pilot study in 10 patients with SSc with 15 DUs, high-frequency ultrasound was found to be a feasible method to measure a range of SSc-related DUs. ${ }^{15}$ Similarly, Sulliman et al. ${ }^{16}$ reported (currently only in abstract form) successful measurement of SSc-related DUs by musculoskeletal ultrasound. In a recent study, ${ }^{17}$ digital planimetry by free hand or fitting a semi-eclipse was found to be a reliable method to measure DU surface area, with good agreement between the two techniques. Baron et al. ${ }^{2}$ reported moderate intra- $(0.57)$ and inter-rater (0.48) reliability for the measurement of DU by surface area in a study using digital callipers.

Our study highlights a number of important lessons for the design of future SSc clinical trials relating to the definition of DUs. First, the development of our proposed DU definitions benefitted from a diverse multi-disciplinary working group, including patient representation. Second, different outcome measures may be needed in preventive studies compared to those of treatments for DUs. In our study, inter-rater reliability was found to be highest in the context of 'studies of treatments for DUs' compared to 'preventive studies' and overall reliability, which could indicate that raters find this classification of lesions (i.e. ulcer vs healed/no ulcer) useful in this context. Third, the role of training to improve the reliability of rater grading warrants investigation. Finally, a number of images had very high or perfect inter-rater agreement (Figure 1), and future studies should consider the production of an 'atlas' of exemplar images to inform DU definitions, both for training purposes and to facilitate standardisation (which could improve inter-rater reliability).

In conclusion, although our proposed DU definitions had high intra-rater reliability, the agreement between raters was poor. Our study provides a number of invaluable insights for the design of future DU clinical trials. Future research is needed to improve the reliability of clinician assessment of SSc-DUs as an outcome measure and explore the complementary roles of objective measurement techniques and PRO instruments in assessing the severity and impact of SSc-DU.

\section{Acknowledgements}

The authors are grateful to all the clinicians (in alphabetical order) who participated in the study but who were not members 
of the working group: Yasmeen Ahmad, Marina Anderson, Eileen Baildem, Theresa Barnes, Maya Buch, Francesco Del Galdo, Emma Derrett-Smith, Frances Hall, Neil McHugh, Simon Meggitt, Catherine H Orteu, Claire Pain, Muditha Samaranayaka, Anita Smyth and Douglas Veale. The authors acknowledge the contributions made by Janet Ravenscroft as a working group member and Vasiliki Tsami in the construction of the web-based interface. The authors would also like to thank Mr Steve Cottrell and colleagues from the Medical Illustration, Salford Royal NHS Foundation Trust, for collecting the images.

\section{Declaration of conflicting interests}

The author(s) declared no potential conflicts of interest with respect to the research, authorship and/or publication of this article.

\section{Funding}

This work was supported by Arthritis Research UK (grant no. 20482).

\section{References}

1. Herrick AL, Roberts C, Tracey A, et al. Lack of agreement between rheumatologists in defining digital ulceration in systemic sclerosis. Arthritis Rheum 2009; 60(3): 878-882.

2. Baron M, Chung L, Gyger G, et al. Consensus opinion of a North American Working Group regarding the classification of digital ulcers in systemic sclerosis. Clin Rheumatol 2014; 33(2): 207-214.

3. Hughes M, Roberts C, Tracey A, et al. Does the clinical context improve the reliability of rheumatologists grading digital ulcers in systemic sclerosis? Arthritis Care Res 2016; 68(9): 1340-1345.

4. Wigley FM, Wise RA, Seibold JR, et al. Intravenous iloprost infusion in patients with Raynaud phenomenon secondary to systemic sclerosis: a multicenter, placebo-controlled, double-blind study. Ann Intern Med 1994; 120(3): 199-206.

5. Matucci-Cerinic M, Denton CP, Furst DE, et al. Bosentan treatment of digital ulcers related to systemic sclerosis: results from the RAPIDS-2 randomised, double-blind, placebo-controlled trial. Ann Rheum Dis 2011; 70(1): 32-38.

6. Hughes M, Ong VH, Anderson ME, et al. Consensus best practice pathway of the UK Scleroderma Study Group: digital vasculopathy in systemic sclerosis. Rheumatology 2015; 54(11): 2015-2024.
7. Hachulla E, Hatron PY, Carpentier P, et al. Efficacy of sildenafil on ischaemic digital ulcer healing in systemic sclerosis: the placebo-controlled SEDUCE study. Ann Rheum Dis 2016; 75(6): 1009-1015.

8. Matucci-Cerinic M, Krieg T, Guillevin L, et al. Elucidating the burden of recurrent and chronic digital ulcers in systemic sclerosis: long-term results from the DUO Registry. Ann Rheum Dis 2016; 75(10): 1770-1776.

9. Gliddon AE, Doré CJ, Black CM, et al. Prevention of vascular damage in scleroderma and autoimmune Raynaud's phenomenon: a multicenter, randomized, double-blind, placebo-controlled trial of the angiotensin-converting enzyme inhibitor quinapril. Arthritis Rheum 2007; 56(11): 38373846.

10. Khanna D, Denton CP, Merkel PA, et al. Effect of macitentan on the development of new ischemic digital ulcers in patients with systemic sclerosis: DUAL-1 and DUAL-2 randomized clinical trials. JAMA 2016; 315(18): 1975-1988.

11. van den Hoogen F, Khanna D, Fransen J, et al. 2013 classification criteria for systemic sclerosis: an American college of rheumatology/European league against rheumatism collaborative initiative. Ann Rheum Dis 2013; 72(11): 17471755.

12. Fleiss JL and Cohen J. The equivalence of weighted kappa and the intraclass correlation coefficient as measures of reliability. Educ Psychol Meas 1973; 33: 613-619.

13. Viera AJ and Garrett JM. Understanding interobserver agreement: the kappa statistic. Fam Med 2005; 37(5): 360 363.

14. Suliman YA, Bruni C, Johnson SR, et al. Defining skin ulcers in systemic sclerosis: systematic literature review and proposed World Scleroderma Foundation (WSF) definition. J Scleroderma and Relat Disord 2017; 2: 115-120.

15. Hughes M, Moore T, Manning J, et al. A pilot study using high-frequency ultrasound to measure digital ulcers: a possible outcome measure in systemic sclerosis clinical trials? Clin Exp Rheumatol 2017; 106(4): 218-219.

16. Suliman Y, Raganath VK, Kafaja S, et al. Novel use of musculoskeletal ultrasound (MSUS) to measure ulcers in the skin of systemic sclerosis (SSc) patients. Arthritis Rheum 2015; 67(Suppl. 1): 2988.

17. Simpson V, Hughes M, Wilkinson J, et al. Quantifying digital ulcers in systemic sclerosis: reliability of computerassisted planimetry in measuring lesion size. Arthritis Care Res (Hoboken) 2017; 70: 486-490. DOI: 10.1002/ acr.23300. 